\title{
Functional renormalization flow and dynamical chiral symmetry breaking of QCD
}

\author{
Ming-Fan L:* and Mingxing Lud \\ Zhejiang Institute of Modern Physics, Zhejiang University, Hangzhou, PR China, 310027
}

\begin{abstract}
The dependence of functional renormalization group equation on regulators is investigated. A parameter is introduced to control the suppression of regulators. Functional renormalization group equations will become regulator-independent if this newly introduced parameter is sent to infinity in the end of calculation. One-loop renormalization flow equations of QCD are derived. The novelty is that both the coupling running equation and the mass running equation are mass-dependent. Different flow patterns are explored. A mechanism for non-occurrence of dynamical chiral symmetry breaking is arrived at. The existence of a conformal window is also discussed in the language of renormalization flow.
\end{abstract}

PACS numbers:

\section{INTRODUCTION}

Renormalization is a concept about connecting physics at different scales. Deriving large scale physics from small scale physics is an important subject, and vice versa. To derive large scale behaviors of QCD from first principle is a long-standing difficult problem.

Functional renormalization group method originates from Wilson's idea of integrating momentum slices successively, and studies how theories flow between different scales. The most commonly used formulations of functional renormalization group include Polchinski's formalism [1] and Wetterich's formalism [2] (for reviews, see for example, [3, 4] ). Functional renormalization group method uses a regulator term to modify propagators, thereby controls contributions of modes of different energy scales to the effective action. In the Wetterich's formalism, for example, the regulator suppresses the low energy modes and lets the high energy modes intact.

There have been many applications of functional remormalization group method, for example, in the analysis of QED [5], Non-Abelian gauge theory [6, 7], phase transitions in gauge theories 8], non-linear diffusion system [9], Dyson's hierarchical model [10]. For reviews of applications in specific areas, see 11 for BCS-BEC corssover, [12] for quark confinement, 13] for nuclear force, [14] for general. Recent applications include, sine-Gordon type models [15, 16], nonrelativistic inverse square potential [17], anharmonic oscillator [18], Ising model [19], etc.

However, the use of the functional renormalization group method is handicapped by one issue. The flow equations derived through this method depend on regulation schemes, thus their physical meanings are ambiguous 20-22]. One bypasses this problem by arguing that flow equations become the true physical flow equations when the cutting scale runs to zero (in the Wetterich's formalism). But one may want not only the behavior

\footnotetext{
*Electronic address: 11006139@zju.edu.cn
}

†Electronic address: luo@zimp.zju.edu.cn at zero energy scale, but also properties at finite energy scales.

In this article, we will investigate this problem closely to see whether we can get a regulator-independent renormalization flow. The idea is to introduce a parameter to control the suppressiveness of a regulator. To make a complete cut, the suppressiveness below the cut-off should be sent to infinity in the end. It is conceivable that the result would be regulator-independent in the infinite suppression limit, since all proper regulators then become the same: a clear cut.

The model that we will use is QCD in $d$-dimensional Euclidian spacetime. Recently, lattice simulations, see 23 27] for example, show that there exists a conformal window for appropriate number of fermions of QCD. Contrary to former anticipations, dynamical chiral symmetry breaking ceases to occur with a lower fermion number than the critical fermion number for asymptotic freedom, $N_{f}^{\ddagger}=11 \times 3 / 2$. The critical fermion number for chiral symmetry breaking is accessed to be in the interval $9<N_{f}^{\dagger}<13$, which is slightly larger than the lower turning point obtained from the two-loop $\beta$-function of $\mathrm{QCD}, 51 \times 3 / 19 \approx 8$.

There have been many tries to explain the existence of this conformal window, for example, by an ansatz of all-loop $\beta$-function [28], mass-dependent $\beta$-functions [29], critical scaling laws [30], etc.

In this article, we will tackle the problem through renormalization flows in the $\tilde{m}^{2}-\tilde{g}^{2}$ plane. Here, $\tilde{m}^{2}$ and $\tilde{g}^{2}$ stand for dimensionless mass and coupling, respectively. We will see that dynamical chiral symmetry breaking can be ceased by and only by an IR-attractive nontrivial fixed point with a finite dimensionless mass in the $\tilde{m}^{2}-\tilde{g}^{2}$ plane. All flows attracted to such a fixed point will have a constant dimensionless mass in the infrared, which means a zero mass in the infrared. Such a fixed point can also exist when there is a nontrivial fixed point in the axis of the coupling of QCD. So, when $N_{f}<11 \times 3 / 2$, dynamical chiral symmetry breaking may not occur and a conformal window can open up.

The structure of this articles is as follows. In section III we briefly review the functional renormalization group method in Wetterich formalism; in section III, we intro- 
duce a parameter to control the suppressiveness of a regulator; in section [V] we apply the method to QCD and derive its mass-dependent one-loop renormalization equations; in section $\nabla$ we prove the regulator-independence of flow equations when the suppressiveness tends to infinity; in section VI we draw flow patterns to gain insights for dynamical chiral symmetry breaking; in section VII. we show that a conformal window can indeed turn up; finally, in section VIII, we give our conclusion.

\section{BRIEF REVIEW OF THE FUNCTIONAL RENORMALIZATION GROUP}

Now we sketch the functional renormalization group method in the Wetterich formalism. To start with, one adds a regulator term $\Delta S_{k}$ to any given action $S$. For a scaler theory, the regulator will have the form,

$$
\Delta S_{k}=\frac{1}{2} \int_{q} \varphi(-q) R_{k}(q) \varphi(q),
$$

where $R_{k}(q)=r\left(q^{2} / k^{2}\right) q^{2}$. It is to suppress the low energy modes in the momentum integration of the effective action, and keep the high energy modes intact. Performing the integration, one is left with a theory of low energy modes. In other words, a theory of all range energy modes is reduced to a low energy effective theory.

For this purpose, there are some requirements on the regulator. Let $k$ be the scale at which one wants to cut off the theory. One first requires that $R_{k}(q) \sim 0$ when $q^{2} \gg$ $k^{2}$. Secondly, one usually takes $R_{k}(q) \sim k^{2}$ for $q^{2} \ll k^{2}$. Effectively, the low energy modes gets a mass suppression of mass $k^{2}$. (Later we will see that this requirement is not enough, which is the source of the regulator-dependence of functional renormalization flow.) To ensure the new theory identical to the original one when the regulator term is removed, one needs also to have $R_{k}(q) \sim 0$ when $k^{2} \rightarrow 0$.

Shown in FIG. 1 is the profile of a typical regulator. Plotted also is $\partial_{t} R_{k}\left(\partial_{t}=k \partial_{k}\right)$, which has a peak at $q^{2} / k^{2}=1$. The peak reflects the fact that the theory is cut at $q^{2}=k^{2}$.

Regulators often used may include exponentials, such as

$$
r\left(q^{2} / k^{2}\right)=\frac{1}{1-e^{-q^{2} / k^{2}}} e^{-q^{2} / k^{2}},
$$

or step-functions, such as,

$$
r\left(q^{2} / k^{2}\right)=\left(k^{2} / q^{2}-1\right) \Theta\left(1-q^{2} / k^{2}\right),
$$

where $\Theta(x)$ is the Heaviside step function.

Let $\Gamma_{k}$ be the $k$-dependent effective action of the low energy effective theory and $\tilde{\Gamma}$ be the effective action of the regulated theory of action $S+\Delta S_{k}$. The flow equation of the effective action (the Wetterich equation) can be derived as,

$$
\partial_{t} \Gamma_{k}=\frac{1}{2} \operatorname{Tr}\left[\left(\partial_{t} R_{k}\right) \tilde{G}_{\varphi \varphi}\right]
$$

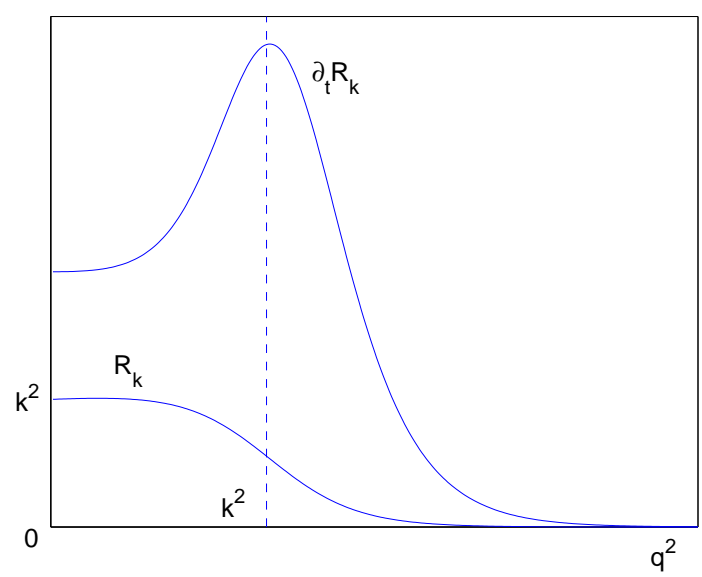

FIG. 1: A typical profile of regulators.

where $\partial_{t}=k \partial_{k} . \tilde{G}_{\varphi \varphi}=\langle\varphi \varphi\rangle-\langle\varphi\rangle\langle\varphi\rangle=\left(\Gamma_{k}^{(2)}+R_{k}\right)^{-1}$, is the connected two-point Green function of the regulated theory.

In this equation, one observes that $\partial_{t} \Gamma_{k}$ depends mainly on modes where $\partial_{t} R_{k}$ peaks. It reflects the fact that the renormalization flow is driven locally and depends only on modes in the vicinity of the cutting scale. Actually this implements Wilson's idea of 'integrating a single momentum slice'.

This equation is a functional equation of $\Gamma_{k}$. It is exact and incorporates all non-perturbative effects. However, it usually cannot be solved exactly. To extract results from this equation, approximations must be performed. A common practice is the method of truncation. In this approach, an ansatz of the form of the effective action $\Gamma_{k}$ is made and the right hand side of the equation (4) is projected onto the ansatz of the effective action. Then this equation is used to derive the flowing of coefficients in the ansatz of the effective action.

\section{REGULATORS}

In this section, a parameter will be introduced to control the suppressiveness of regulators.

As has been mentioned above, one usually requires $R_{k}(q) \sim k^{2}$ for $q^{2} \ll k^{2}$. The suppression with a mass $k^{2}$ may not be enough. Instead, we would like to replace it with $R_{k}(q) \sim s^{2} k^{2}$. The parameter $s^{2}$ controls to what degree the low energy modes are suppressed. To completely exclude the effect of low energy modes, we should let $s^{2} \rightarrow \infty$ in the end of calculations.

Now we are to write down a typical regulator. An immediate choice may be a step-type regulator, due to its simplicity,

$$
r\left(q^{2} / k^{2} ; s^{2}\right)=\left(s^{2} k^{2} / q^{2}-1\right) \Theta\left(1-q^{2} / k^{2}\right) .
$$

However this regulator is not continuous, and can raise problems. So we need for continuous ones. There is a lot 


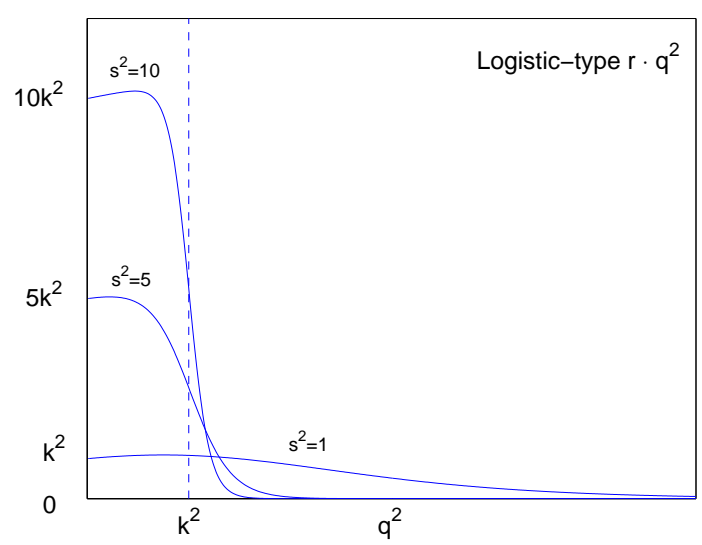

FIG. 2: Logistic-type regulators with different values of $s^{2}$.

of smooth approximations of the Heaviside step function $\Theta(x)$, so we can use these functions to construct smooth regulators. For example, a logistic-type regulator,

$$
r\left(q^{2} / k^{2} ; s^{2}\right)=\frac{1}{1-e^{-q^{2} /\left(s^{2} k^{2}\right)}} \frac{1+e^{-s^{2}}}{1+e^{-s^{2}\left(1-q^{2} / k^{2}\right)}} ;
$$

an erf-type regulator,

$$
r\left(q^{2} / k^{2} ; s^{2}\right)=\frac{s^{2} k^{2}}{q^{2}} \frac{1+\operatorname{erf}\left(s^{2}\left(1-q^{2} / k^{2}\right)\right)}{1+\operatorname{erf}\left(s^{2}\right)} ;
$$

and an arctan-type regulator,

$r\left(q^{2} / k^{2} ; s^{2}\right)=\frac{s^{2} k^{2}}{q^{2}} e^{-q^{2} / k^{2}} \cdot \frac{\pi / 2+\arctan \left(s^{2}\left(1-q^{2} / k^{2}\right)\right)}{\pi / 2+\arctan \left(s^{2}\right)}$.

In all cases, the second factor is a smooth approximation of the step function $\Theta\left(1-q^{2} / k^{2}\right)$ which is arrived at the limit $s^{2} \rightarrow \infty$. The first factor is to ensure $r\left(q^{2} / k^{2} ; s^{2}\right) \sim s^{2} k^{2} / q^{2}$ when $q^{2} \ll k^{2}$. When $s^{2} \rightarrow \infty$, for all above cases, $r\left(q^{2} / k^{2} ; s^{2}\right) \cdot q^{2}$ tend to an infinity step function which takes the value of infinity at $q^{2}<k^{2}$ and the value of zero at $q^{2}>k^{2}$. One can see this trend in FIG. 2. where we have plotted profiles of the logistic-type regulator with different values of $s^{2}$.

Actually, the trend of tending to a step function and the trend of tending to infinite suppression in the left and no suppression in the right can always be parameterized by two independent parameters. However, we use only one parameter $s^{2}$, since our later discussions do not depend on this simplicity assumption.

To implement numerical computations, we will take a folded-straightline regulator, in the form

$$
r\left(q^{2} / k^{2} ; s^{2}\right) \cdot q^{2} / k^{2}=\xi_{0}-\frac{\xi_{0}}{\varepsilon}\left(\frac{q^{2}}{k^{2}}-1\right)
$$

in the interval $[1-\varepsilon, 1+\varepsilon]$, and constant elsewhere. $s^{2} \rightarrow$ $\infty$ is equivalent to $\xi_{0} \rightarrow \infty$ and $\varepsilon \rightarrow 0$. The order of taking limit is irrelevant.

\section{APPLICATION TO QCD}

In this section we will apply the functional renormalization group method to QCD to study its renormalization flow.

The Lagrangian of QCD in Euclidian spacetime is, 31]

$$
\begin{aligned}
\mathfrak{L}_{E}= & \bar{\psi}^{a_{f}}\left(i \not D+m_{a_{f}}\right) \psi^{a_{f}}+\frac{1}{4}\left(F_{\mu \nu}^{a}\right)^{2} \\
& -\frac{1}{2 \xi}\left(\partial^{\mu} A_{\mu}^{a}\right)^{2}+\bar{c}^{a} \partial^{\mu} D_{\mu}^{a c} c^{c} \\
= & \bar{\psi}^{a_{f}}\left(i \gamma^{\mu} \partial_{\mu}+m_{a_{f}}\right) \psi^{a_{f}}+g \bar{\psi}^{a_{f}} \gamma^{\mu} t^{a} A_{\mu}^{a} \psi^{a_{f}} \\
- & \frac{1}{2} A_{\mu}^{a} \partial^{2} A_{\mu}^{a}+\bar{c}^{a} \partial^{2} c^{a}+g f^{a b c} \bar{c}^{a} \partial_{\mu}\left(A_{\mu}^{b} c^{c}\right) \\
+ & g f^{a b c}\left(\partial_{\kappa} A_{\lambda}^{a}\right) A_{\kappa}^{b} A_{\lambda}^{c}+\frac{1}{4} g^{2} f^{a b e} f^{c d e} A_{\kappa}^{a} A_{\lambda}^{b} A_{\kappa}^{c} A_{\left(l_{1} .0\right)}
\end{aligned}
$$

We have used the convention $\left\{\gamma^{\mu}, \gamma^{\nu}\right\}=-2 \delta^{\mu \nu} I_{4 \times 4}$ and the gauge $\xi=1$.

Now go to momentum space and take the following ansatz for the effective action

$$
\begin{aligned}
\Gamma & =\int_{p} Z_{\psi} \bar{\psi}^{a_{f}}(p)(p+m) \psi^{a_{f}}(p) \\
& +\frac{1}{2} Z_{A} \int_{p} A_{\mu}^{a}(-p)\left(p^{2} \delta^{\mu \nu}-\left(1-\frac{1}{\xi}\right) p^{\mu} p^{\nu}\right) A_{\nu}^{a}(p) \\
& -Z_{c} \int_{p} \bar{c}^{a}(p) p^{2} c^{a}(p) \\
& +g Z_{\psi} \sqrt{Z_{A}} \int_{p} \int_{p^{\prime}} \bar{\psi}^{a_{f}}(p) \gamma^{\mu} t^{a} A_{\mu}^{a}\left(p-p^{\prime}\right) \psi^{a_{f}}\left(p^{\prime}\right) \\
& +g Z_{c} \sqrt{Z_{A}} \int_{p} \int_{p^{\prime}} f^{a b c}\left(i p_{\mu}\right) \bar{c}^{a}(p) A_{\mu}^{b}\left(p-p^{\prime}\right) c^{c}\left(p^{\prime}\right) \\
& +g Z_{A} \sqrt{Z_{A}} \int_{p} \int_{p^{\prime}} \int_{q}(2 \pi)^{d} \delta^{(d)}\left(p+p^{\prime}+q\right) \\
& \quad \cdot f^{a b c}\left(-i q_{\mu}\right) A_{\nu}^{a}(q) A_{\mu}^{b}(p) A_{\nu}^{c}\left(p^{\prime}\right) \\
+ & \frac{1}{4} g^{2} Z_{A}^{2} \int_{p} \int_{p^{\prime}} \int_{q} \int_{q^{\prime}}(2 \pi)^{d} \delta^{(d)}\left(p+p^{\prime}+q+q^{\prime}\right) \\
& \quad \cdot f^{a b e} f^{c d e} A_{\kappa}^{a}(p) A_{\lambda}^{b}\left(p^{\prime}\right) A_{\kappa}^{c}(q) A_{\lambda}^{d}\left(q^{\prime}\right) .
\end{aligned}
$$

For simplicity, we have assumed all fermions have the same mass.

The regulators are

$$
\begin{aligned}
\Delta S_{\psi} & =\int_{p} \int_{q} \bar{\psi}^{a_{f}}(p) \hat{R}_{\psi}^{a_{f} b_{f}}(p, q) \psi^{b_{f}}(q) \\
\Delta S_{A} & =\frac{1}{2} \int_{p} \int_{q} A_{\mu}^{a}(-p) \hat{R}_{A}^{a b, \mu \nu}(p, q) A_{\nu}^{b}(q) \\
\Delta S_{c} & =\int_{p} \int_{q} \bar{c}^{a}(p) \hat{R}_{c}^{a b}(p, q) c^{b}(q),
\end{aligned}
$$

with

$$
\begin{aligned}
\hat{R}_{\psi}^{a_{f} b_{f}}(p, q) & =Z_{\psi} \delta^{a_{f} b_{f}} \delta_{p q} r_{\psi}\left(p^{2} / k^{2} ; s^{2}\right) p \\
\hat{R}_{A}^{a b, \mu \nu}(p, q) & =Z_{A} \delta^{a b} \delta^{\mu \nu} \delta_{p q} r_{A}\left(p^{2} / k^{2} ; s^{2}\right) p^{2}, \\
\hat{R}_{c}^{a b}(p, q) & =-Z_{c} \delta^{a b} \delta_{p q} r_{A}\left(p^{2} / k^{2} ; s^{2}\right) p^{2}
\end{aligned}
$$


with $\left(1+r_{\psi}\right)^{2}=\left(1+r_{A}\right)$.

The functional renormalization group equation can be derived as

$$
\begin{aligned}
\partial_{t} \Gamma & =\frac{1}{2} \operatorname{Tr}\left[\left(\partial_{t} \hat{R}_{A}\right) \tilde{G}_{A A}\right]-\operatorname{Tr}\left[\left(\partial_{t} \hat{R}_{\psi}\right) \tilde{G}_{\psi \bar{\psi}}\right] \\
& -\operatorname{Tr}\left[\left(\partial_{t} \hat{R}_{c}\right) \tilde{G}_{c \bar{c}}\right]
\end{aligned}
$$

where $\tilde{G}_{A A}, \tilde{G}_{\psi \bar{\psi}}$ and $\tilde{G}_{c \bar{c}}$ are connected Green functions,

$$
\begin{aligned}
\tilde{G}_{A A} & =\langle A(q) A(p)\rangle-\langle A(q)\rangle\langle A(p)\rangle, \\
\tilde{G}_{\psi \bar{\psi}} & =\langle\psi(q) \bar{\psi}(p)\rangle-\langle\psi(q)\rangle\langle\bar{\psi}(p)\rangle, \\
\tilde{G}_{c \bar{c}} & =\langle c(q) \bar{c}(p)\rangle-\langle c(q)\rangle\langle\bar{c}(p)\rangle .
\end{aligned}
$$

Since $\tilde{G} \cdot \tilde{\Gamma}^{(2)}=1$, one has

$$
\begin{aligned}
\tilde{G}_{\psi \bar{\psi}}= & {\left[\tilde{\Gamma}_{\bar{\psi} \psi}^{(2)}-\tilde{\Gamma}_{\bar{\psi} A}^{(2)} \tilde{\Gamma}_{A A}^{(2)-1} \tilde{\Gamma}_{A \psi}^{(2)}\right]^{-1} } \\
\tilde{G}_{A A}= & {\left[\tilde{\Gamma}_{A A}^{(2)}-\tilde{\Gamma}_{A c}^{(2)} \tilde{\Gamma}_{\bar{c} c}^{(2)-1} \tilde{\Gamma}_{\bar{c} A}^{(2)}-\tilde{\Gamma}_{A \bar{c}}^{(2)} \tilde{\Gamma}_{c \bar{c}}^{(2)-1} \tilde{\Gamma}_{c A}^{(2)}\right.} \\
& \left.-\tilde{\Gamma}_{A \psi}^{(2)} \tilde{\Gamma}_{\bar{\psi} \psi}^{(2)-1} \tilde{\Gamma}_{\bar{\psi} A}^{(2)}-\tilde{\Gamma}_{A \bar{\psi}}^{(2)} \tilde{\Gamma}_{\psi \bar{\psi}}^{(2)-1} \tilde{\Gamma}_{\psi A}^{(2)}\right]^{-1}, \\
\tilde{G}_{c \bar{c}}= & {\left[\tilde{\Gamma}_{\bar{c} c}^{(2)}-\tilde{\Gamma}_{\bar{c} A}^{(2)} \tilde{\Gamma}_{A A}^{(2)-1} \tilde{\Gamma}_{A c}^{(2)}\right]^{-1} }
\end{aligned}
$$

After a lengthy calculation, we arrived at the following flow equations for the coupling $g$ and the mass $m$ (see Appendix $\mathrm{A}$ for the details)

$$
\begin{aligned}
\frac{\partial_{t} m}{m} & =-\tilde{g}^{2} C_{2}(r) K_{m} \\
\frac{\partial_{t} g}{g} & =-\tilde{g}^{2}\left[C_{2}(r) K_{1}+C_{2}(G) K_{2}-N_{f} C(r) K_{3}\right]
\end{aligned}
$$

Dimensionless quantities are defined as $\tilde{m}=m / k, \tilde{g}^{2}=$ $\left[\int d \Omega_{d} /(2 \pi)^{d}\right] g^{2} / k^{4-d}$ and

$$
\begin{aligned}
K_{m} & =d\left[J\left(1,4,1 ; \tilde{m}^{2}\right)+J\left(0,2,2 ; \tilde{m}^{2}\right)\right] \\
& -\frac{(d-1)(d-2)}{d} J\left(1,3,1 ; \tilde{m}^{2}\right) \\
K_{1} & =(d-2)\left[J\left(1,4,1 ; \tilde{m}^{2}\right)+J\left(0,2,2 ; \tilde{m}^{2}\right)\right] \\
& -\frac{4(d-2)}{d} J\left(-1,0,3 ; \tilde{m}^{2}\right)-\frac{(d-1)(d-2)}{d} J\left(1,3,1 ; \tilde{m}^{2}\right. \\
K_{2} & =-\frac{d-2}{2}\left[J\left(1,4,1 ; \tilde{m}^{2}\right)+J\left(0,2,2 ; \tilde{m}^{2}\right)\right] \\
& +\frac{2(d-2)}{d} J\left(-1,0,3 ; \tilde{m}^{2}\right) \\
& +\frac{3(d-1)}{d} J\left(1,5,1 ; \tilde{m}^{2}\right)+\frac{2(d-1)}{d} J\left(0,3,2 ; \tilde{m}^{2}\right) \\
& -\left[\frac{16(d-2)}{d(d+2)}+\frac{d-14}{2}+\frac{8}{d}\right] J\left(2,6,0 ; \tilde{m}^{2}\right) ; \\
K_{3} & =-\frac{8}{d} J\left(-1,-2,3 ; \tilde{m}^{2}\right)+\frac{16(d+4)}{d(d+2)} J\left(-2,-4,4 ; \tilde{m}^{2}\right) \\
& -\frac{64}{d(d+2)} J\left(-3,-6,5 ; \tilde{m}^{2}\right) .
\end{aligned}
$$

Here $J\left(a, b, c ; \tilde{m}^{2}\right)$ are dimensionless momentum integrals defined by

$$
J\left(a, b, c ; \tilde{m}^{2}\right) \equiv \int_{l} \frac{\left(\partial_{t} r_{A}\right) \cdot k^{2(a+c)-d}(2 \pi)^{d} / \int d \Omega_{d}}{\left(l^{2}\right)^{a}\left(1+r_{\psi}\right)^{b}\left[m^{2}+l^{2}\left(1+r_{\psi}\right)^{2}\right]^{c}}
$$

This integral encodes all regulator-relating information. It will be investigated closely in the next section.

\section{MOMENTUM INTEGRAL AND REGULATOR-INDEPENDENCE}

Since the Wetterich equation has a one-loop structure, momentum integrals appearing in flow equations all have the form of (27), provided there is only one mass parameter in the considered theory.

Generally the following relations hold,

$$
\begin{aligned}
& J\left(a, b, c ; \tilde{m}^{2}\right) \\
& =\tilde{m}^{2} J\left(a, b, c+1 ; \tilde{m}^{2}\right)+J\left(a-1, b-2, c+1 ; \tilde{m}^{2}(28)\right. \\
& \frac{d J\left(a, b, c ; \tilde{m}^{2}\right)}{d \tilde{m}^{2}}=-c J\left(a, b, c+1 ; \tilde{m}^{2}\right) .
\end{aligned}
$$

First consider the integrals with $a+c=d / 2$. The integration can be easily carried out analytically since $k \partial_{k} r_{A}=-l \partial_{l} r_{A}$. When $\tilde{m}^{2}=0$ and $b / 2+c-1>0$, the result is

$$
J(a, b, c ; 0)=\frac{1}{\frac{b}{2}+c-1} .
$$

When $\tilde{m}^{2} \in[0,1)$ and $b / 2+c-1>0$, the result can be given by the series,

$$
J\left(a, b, c ; \tilde{m}^{2}\right)=\sum_{n=0}^{\infty} \frac{(c)_{n}}{n !} J(a, b, c+n ; 0)\left(-\tilde{m}^{2}\right)^{n},
$$

where $(c)_{n}$ is the rising factorial, defined as $(c)_{0}=1$, $(c)_{1}=c,(c)_{n}=c(c+1) \ldots(c+n-1)$. In this situation, since the momentum integral can be carried out analytically without any reference to the concrete form of the 2 regulator, these results are regulator-independent.

The integrals in the flow equations (25) and (26) are all of this kind when $d=4$. When $\tilde{m}^{2}=0$, the $K$ coefficients can be calculated as:

$$
K_{m}=3, \quad K_{1}=0, \quad K_{2}=\frac{11}{3 \times 2}, \quad K_{3}=\frac{4}{3 \times 2} .
$$

The ordinary one-loop $\beta$ function of QCD and the ordinary mass running equation are reproduced.

The integrals with $a+c \neq d / 2$ are little more complicated, though generally the integration can not be carried out analytically. As we will eventually take limit after integration, we can make an approximation for the integral. As has been aforementioned, the flow is driven only by modes in the vicinity of the cutoff scale, as indicated by 
the fact that $\partial_{t} r_{A}$ peaks at $q^{2} / k^{2}=1$. Actually the peak of the integrand is rather sharp, and values at elsewhere are suppressed to nearly zero if $s^{2}$ is sufficiently large. So we can make the approximation:

$$
\int_{0}^{\infty} d \tilde{l}^{2} \cdot \text { integrand } \approx \int_{1-\varepsilon}^{1+\varepsilon} d \tilde{l}^{2} \cdot \text { integrand. }
$$

When $s^{2} \rightarrow \infty, \varepsilon \rightarrow 0$.

The integral $J(a, b, c ; 0)$ can be evaluated as:

$$
\int_{\tilde{l}^{2}=1-\varepsilon}^{\tilde{l}^{2}=1+\varepsilon} l^{d-1} d l \frac{\partial_{t} r_{A} \cdot k^{2(a+c)-d}}{\left(l^{2}\right)^{a+c}\left(1+r_{A}\right)^{b / 2+c}} .
$$

When $a+c-d / 2=0$ and $b / 2+c-1>0$, this integral can be carried out directly, yielding $1 /(b / 2+c-1)$ if $s^{2} \rightarrow \infty$ in the end, which is the same result as derived formerly. When $a+c-d / 2 \neq 0$ and $b / 2+c-1>0$, this integral can also be done through the mean value theorem of integration, yielding the same result $1 /(b / 2+c-1)$ if $s^{2} \rightarrow \infty$ in the end. These results are both independent of the specific form of the regulator $r_{A}\left(q^{2} / k^{2} ; s^{2}\right)$, hence regulator-independent.

\section{FLOW PATTERNS AND CHIRAL SYMMETRY BREAKING}

In this section, we first explore different kinds of flow patterns. A mechanism for the restoration of chiral symmetry will be arrived at. Then we prove this mechanism indeed applies to QCD. In this section the discussions will be constrained to situations of $d=4$.

First consider the flow patterns of the one-loop renormalization group of QCD, (25) and (26). The coefficient of $\tilde{g}^{2}$ in the equation (26) always has a zero only if $N_{f}>11 \times 3 / 2$. While $K_{m}$ is always positive, so there is no non-trivial fixed-point for the flow equations (25) and (26). Shown in FIG. 3 are their flow patterns. The left panel is typical for $N_{f}<11 \times 3 / 2$, and the right panel is typical for $N_{f}>11 \times 3 / 2$.

These flow patterns do not capture the non-occurrence of dynamical chiral symmetry breaking. However there are other kinds of flow patterns that may do so. To explore these ones, it is natural to add in two loop contributions as the next step.

Let's recall the ordinary two-loop renormalization group equation for the mass [32],

$$
\partial_{t} \tilde{m}^{2}=-2 \tilde{m}^{2}\left(1+h_{1} \tilde{g}^{2}+h_{2}\left(\tilde{g}^{2}\right)^{2}\right),
$$

with

$$
\begin{aligned}
& h_{1}=3 C_{2}(r), \\
& h_{2}=\frac{3}{4} C_{2}(r)^{2}+\frac{97}{12} C_{2}(r) C_{2}(G)-\frac{5}{6} C_{2}(r) N_{f} .
\end{aligned}
$$

Observe that, in $h_{2}$ the coefficient before $N_{f}$ is negative. So for large enough $N_{f}$, this term will dominate and the
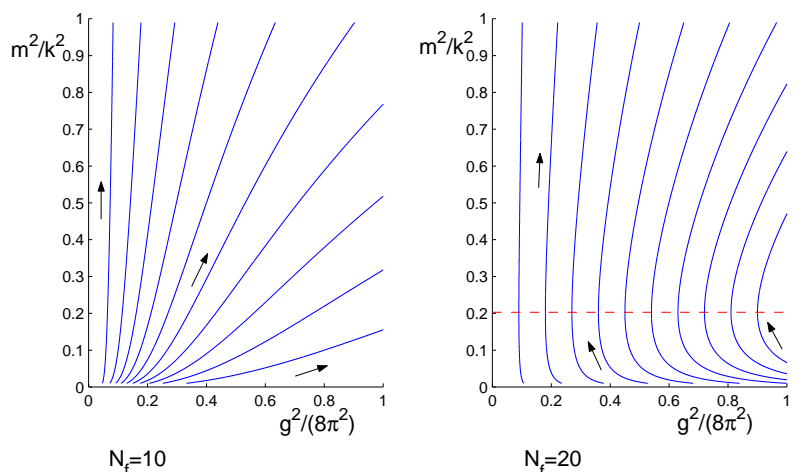

FIG. 3: Flow patterns with $d=4$ and complete suppression. The arrows point to infrared. The red dashed line stands for $\beta=0$.

flow equation of the mass will develop a zero point. In turn, the $\tilde{m}^{2}-\tilde{g}^{2}$ flows will develop a nontrivial fixed point. However, coefficients in this equation are independent of $\tilde{m}^{2}$. In other words, this equation corresponds to Eq. (25) with $\tilde{m}^{2}=0$ but at two-loop. If we use it directly to depict the flows, the fixed point will be displaced and streamlines distorted. Even worse, the flow patterns may be changed. Nevertheless, to see how flow patterns capture the non-occurrence of dynamical chiral symmetry breaking, it will be enough to make a parametrization of Eq. (34).

Literally, we multiply two-loop terms in Eq. (34) by factors $\beta_{i}^{\alpha_{i}} /\left(\tilde{m}^{2}+\beta_{i}\right)^{\alpha_{i}}\left(\alpha_{i}\right.$ and $\beta_{i}$ are constant parameters), and add them to Eq. (25). This form of mass dependence is motivated by the $J$-integral (27). $\quad \alpha_{i}$ is roughly the number of fermion propagators in two loop momentum integrals. The difference among $\beta_{i}$ can be generated after loop momentum integration.

The resulting equation of these manipulations will be used to explore different flow patterns. One can see that the resulting equation may not be the true one of $\mathrm{QCD}$, but it is enough for qualitative investigation.

Concretely, the following system will be considered:

$$
\begin{aligned}
\frac{\partial_{t} \tilde{m}^{2}}{2 \tilde{m}^{2}} & =-\left[1+\tilde{g}^{2} C_{2}(r) K_{m}+H_{2}\left(\tilde{m}^{2}\right)\left(\tilde{g}^{2}\right)^{2}\right] \\
\frac{\partial_{t} \tilde{g}^{2}}{2 \tilde{g}^{2}} & =-\tilde{g}^{2}\left[C_{2}(r) K_{1}+C_{2}(G) K_{2}-N_{f} C(r) K_{3}\right](3)
\end{aligned}
$$

with

$$
H_{2}\left(\tilde{m}^{2}\right)=h_{21} \rho_{1}^{\alpha_{1}}+h_{22} \rho_{2}^{\alpha_{2}}-h_{23} \rho_{3}^{\alpha_{3}},
$$

where

$$
\begin{aligned}
h_{21} & =\frac{97}{12} C_{2}(r) C_{2}(G), \\
h_{22} & =\frac{3}{4} C_{2}(r)^{2}, \\
h_{23} & =\frac{5}{6} C_{2}(r) N_{f},
\end{aligned}
$$




$$
\rho_{i}=\frac{\beta_{i}}{\tilde{m}^{2}+\beta_{i}}, \quad i=1,2,3 .
$$

This system is not the one of QCD. However, since our purpose is to explore different kinds of flow patterns rather than to discuss QCD directly, it is reasonable to use such a system. Actually, to explore different kinds of flow patterns, any functions are permissible in the right hand of the flow equations (37) and (38). The only problem is whether they can lead to new and meaningful flow patterns.

One loop flow equations of QCD, (25) and (26), do not capture the restoration of chiral symmetry, but they provide a base to be perturbed and to generate new flow equations.

Besides the trivial fixed point $\left(\tilde{m}^{2}=0, \tilde{g}^{2}=0\right)$, the flow equations (37) and (38) now may have a nontrivial fixed point. Taking $\tilde{m}^{2}$ as the argument, the possible nontrivial fixed point is

$$
\begin{aligned}
N_{f}^{*} & =\frac{C_{2}(r) K_{1}+C_{2}(G) K_{2}}{C(r) K_{3}}, \\
\tilde{g}^{2 *} & =\frac{-C_{2}(r) K_{m}-\sqrt{\left[C_{2}(r) K_{m}\right]^{2}-4 H_{2}}}{2 H_{2}} .
\end{aligned}
$$

Another unphysical root has been discarded. To ensure $\tilde{g}^{2 *}>0$, the sufficient and necessary condition is:

$$
H_{2}\left(\tilde{m}^{2 *}\right)<0 .
$$

To see the behavior of flows near the above fixed point, we estimate the critical exponents. If $\lambda_{1}$ and $\lambda_{2}$ are the critical exponents of this fixed point, then,

$$
\begin{aligned}
\lambda_{1} \lambda_{2} \approx & {\left[-1 / \tilde{g}^{2 *}+H_{2}\left(\tilde{m}^{2 *}\right)\right]\left[\left.\tilde{g}^{2 *} N_{f} C(r) \frac{d K_{3}}{d \tilde{m}^{2}}\right|_{\tilde{m}^{2 *}}\right]>0 } \\
\lambda_{1}+\lambda_{2} & =-2 \tilde{m}^{2 *}\left[\left.\tilde{g}^{2 *} C_{2}(r) \frac{d K_{m}}{d \tilde{m}^{2}}\right|_{\tilde{m}^{2 *}}+\left.\frac{d H_{2}}{d \tilde{m}^{2}}\right|_{\tilde{m}^{2 *}} \cdot\left(\tilde{g}^{2 *}\right)^{2}\right] \\
& \approx-\left.2 \tilde{m}^{2 *} \frac{d H_{2}}{d \tilde{m}^{2}}\right|_{\tilde{m}^{2 *}} \cdot\left(\tilde{g}^{2 *}\right)^{2} \\
& \propto-\left.\frac{d H_{2}}{d \tilde{m}^{2}}\right|_{\tilde{m}^{2 *}} .
\end{aligned}
$$

When

$$
\left.\frac{d H_{2}}{d \tilde{m}^{2}}\right|_{\tilde{m}^{2 *}}<0(>0),
$$

the fixed point is IR-attractive (IR-repulsive).

One sees that the fixed-point structure of Eqs. (37) and (38) depends on the choice of parameters $\left(\alpha_{i}, \beta_{i}\right)$. For example, the choice of $\left(\alpha_{1}, \alpha_{2}, \alpha_{3}, \beta_{1}, \beta_{2}, \beta_{3}\right)=$ $(3,4,5,1,1,36)$ will lead to an IR-attractive nontrivial fixed point for all $N_{f}>17.12$. While the choice of $(2,3,4,1,2,5)$ will result in an IR-repulsive fixed point for all $N_{f}>25.56$. We have depicted some flow patterns in FIG. 4- FIG. 6,

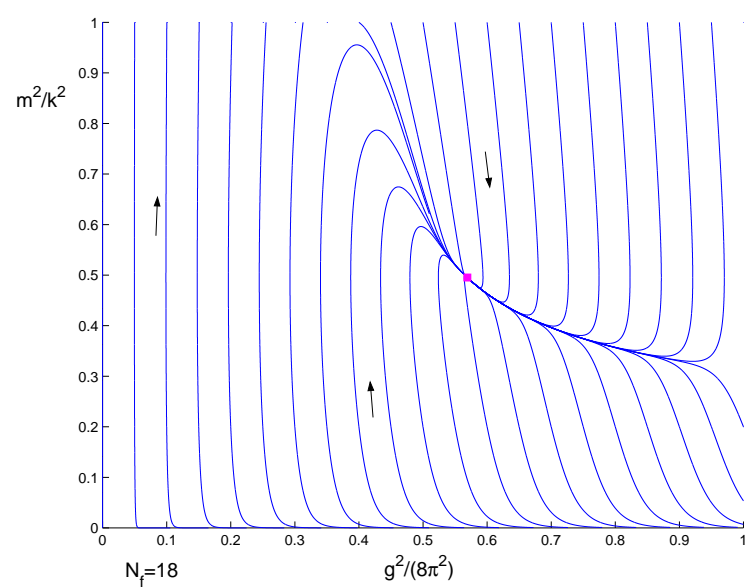

FIG. 4: IR-attractive nontrivial fixed point. The arrows point to infrared. $\left(\alpha_{i}, \beta_{i}\right)=(3,4,5,1,1,36)$.

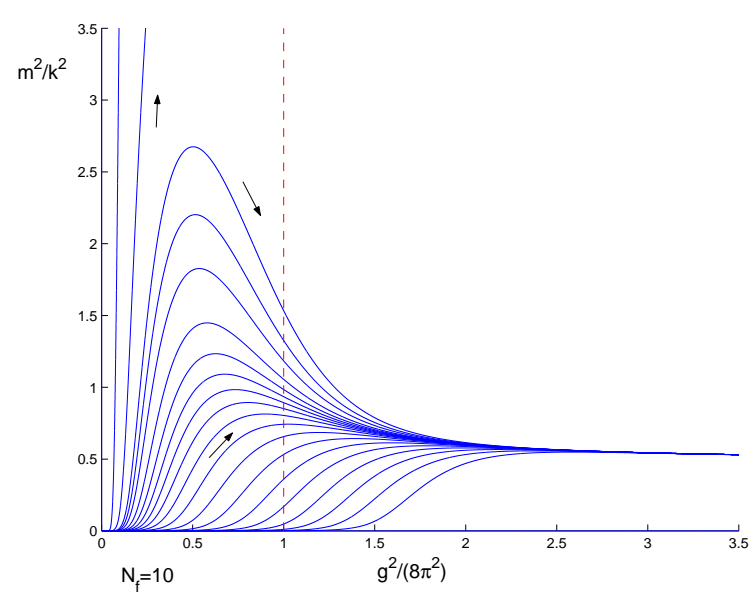

FIG. 5: No nontrivial fixed point. The arrows point to infrared. $\left(\alpha_{i}, \beta_{i}\right)=(3,4,5,1,1,36)$.

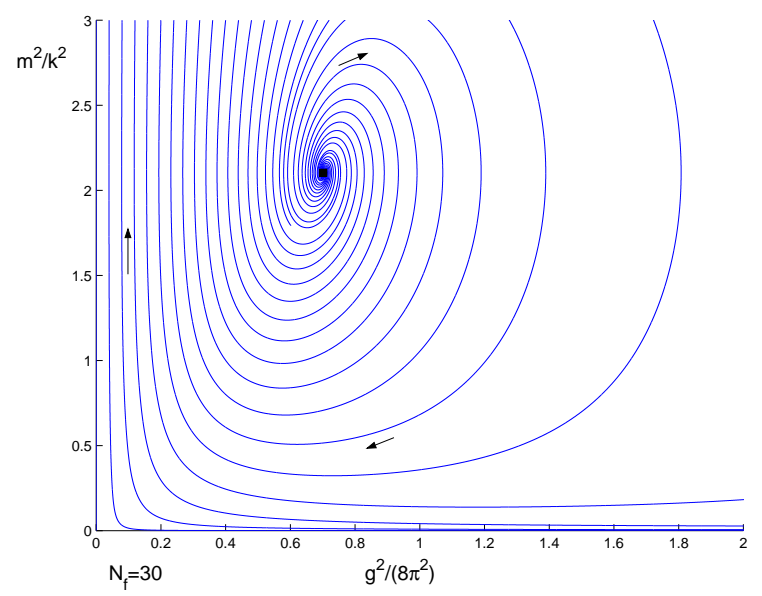

FIG. 6: IR-replusive nontrivial fixed point. The arrows point to infrared. $\left(\alpha_{i}, \beta_{i}\right)=(2,3,4,1,2,5)$. 
Shown in FIG. 4 is the flow pattern for $N_{f}=18$ with $\left(\alpha_{i}, \beta_{i}\right)=(3,4,5,1,1,36)$. One sees that all flows are going to the IR-attractive fixed point. At this point, $\tilde{m}^{2}$ is a constant, which means $m^{2}=\tilde{m}^{2} k^{2}$ goes to zero when $k^{2} \rightarrow 0$. So there is no mass generation or chiral symmetry breaking.

Dynamical chiral symmetry breaking is in principle a non-perurbative behavior, much the same as color confinement. However, color confinement can be gauged via perturbative calculation, in the form of asymptotic freedom. Similarly, dynamical chiral symmetry breaking may also be gauged via perturbative calculation. To chiral symmetry the fermion mass is more relevant than the gauge coupling. So the effect of fermion mass seems more important than higher order quantum corrections. Thus the above discussion from the perspective of perturbative renormaliztion flow is meaningful.

Shown in FIG. [5] is the flow pattern for $N_{f}=10$ with $\left(\alpha_{i}, \beta_{i}\right)=(3,4,5,1,1,36)$. There is no nontrivial fixed point and all flows to the limit $\left(\infty, \tilde{m}^{2}\left(H_{2}=0\right)\right)$. This cannot be interpreted as a restoration of chiral symmetry, since the coupling $\tilde{g}^{2}$ has gone beyond the perturbative region. Shown in FIG. 6 is the flow pattern for $N_{f}=30$ with $\left(\alpha_{i}, \beta_{i}\right)=(2,3,4,1,2,5)$. An IR-repulsive nontrivial fixed point turns up.

In the above, different flow patterns are displayed. One of them, in FIG. 4. provides a mechanism to explain the non-occurrence of dynamical chiral symmetry breaking. Theories attracted to an IR-attractive nontrivial fixed point of a finite $\tilde{m}^{2 *}$ will show no dynamical chiral symmetry breaking.

Now we show this mechanism can also apply to QCD. We shall prove the inverse proposition: a non-occurrence of dynamical chiral symmetry breaking indicates the theory is attracted to an IR-attractive nontrivial fixed point of a finite $\tilde{m}^{2 *}$.

The mass running equation of all-loop QCD can be written as

$$
\partial_{t} \tilde{m}^{2}=-2 \tilde{m}^{2}\left[1+\sum_{i=1}^{\infty} H_{i}\left(\tilde{m}^{2} ; N_{f}\right)\left(\tilde{g}^{2}\right)^{i}\right]
$$

Here $H_{i}\left(\tilde{m}^{2} ; N_{f}\right)$ is the mass-dependent coefficient of the $i$-loop correction to the fermion mass in QCD. Restoration of chiral symmetry requires $m^{2}=0$ in the infrared. So the scaling exponent of $\tilde{m}^{2}$ is smaller than 2 , or the value of sum of terms in the square bracket is smaller than 1. This is possible, because when $N_{f}$ large enough, some of $H_{i}\left(\tilde{m}^{2} ; N_{f}\right)$ will be negative. Since $H_{i}\left(\tilde{m}^{2} ; N_{f}\right)$ are loop corrections to the fermion mass, they will all tend to 0 when $\tilde{m}^{2} \rightarrow \infty$ because of the fermion propagators in the loop integrals. Actually when $\tilde{m}^{2}$ large enough and $\tilde{g}^{2}$ small enough, the sum of quantum corrections will be positive. This is due to the fact: loop integrals with more than one fermion propagators then will subdominate, and the one-loop contribution will dominate.

This discussion can also apply to the non-perturbative situation. Loop integrals with more than one fermion propagators sub-dominate only if $\tilde{m}^{2}$ is sufficiently large. The difference is that the one-loop integral is now with a gluon propagator that has been renormalized in pure gauge theory. It is still positive, for a gluon cannot change to a ghost by mere renormalization. One can see that the above discussion is not based on perturbative expansion, but on $1 / \tilde{m}^{2}$ expansion.

So, if there is no dynamical chiral symmetry breaking, $\tilde{m}^{2}$ should be a finite value in the infrared. The coupling $\tilde{g}^{2}$ should be a nonzero (finite) value as well. The theory is attracted to an IR-attractive nontrivial fixed point of a finite $\tilde{m}^{2 *}$. Thus we proved the proposition.

\section{THE CONFORMAL WINDOW}

In this section, we will see how conformal window is reflected in renormalization flows.

Recall the ordinary two-loop running equation of the coupling in QCD:

$$
\partial_{t} \tilde{g}^{2}=-\tilde{g}^{2}\left(f_{1} \tilde{g}^{2}+f_{2}\left(\tilde{g}^{2}\right)^{2}\right),
$$

with

$$
\begin{aligned}
& f_{1}=\frac{11}{3} C_{2}(G)-\frac{4}{3} C(r) N_{f} \\
& f_{2}=\frac{17}{3} C_{2}(G)^{2}-\frac{10}{3} C_{2}(G) C(r) N_{f}-2 C_{2}(r) C(r) N(50)
\end{aligned}
$$

One can do similar manipulations as those for the mass running equation, however there is another choice which is simpler and more certain.

In Eq. (48), there are two turning points for $N_{f}$ at which the behavior of $\beta_{\tilde{g}^{2}}\left(=\partial_{t} \tilde{g}^{2}\right)$ changes. $\beta_{\tilde{g}^{2}}$ is always positive for $N_{f}>11 \times 3 / 2$ and always negative for $N_{f}<$ $51 \times 3 / 19$. When $51 \times 3 / 19<N_{f}<11 \times 3 / 2$, the sign of $\beta_{\tilde{g}^{2}}$ changes at a nontrivial fixed point, $\tilde{g}^{2 *}=-f_{1} / f_{2}$.

This property can also be found in the one-loop $\beta$ function of $d$-dimensional QCD with $d=4-\epsilon<4$ :

$$
\partial_{t} \tilde{g}^{2}=-\tilde{g}^{2}\left(\epsilon+\tilde{g}^{2} F_{1}(0)\right),
$$

where $F_{1}(0)$ is the value of $2\left[C_{2}(r) K_{1}+C_{2}(G) K_{2}-\right.$ $\left.N_{f} C(r) K_{3}\right]$ with $\tilde{m}^{2}=0$. When $N_{f}<\left[C_{2}(r) K_{1}+\right.$ $\left.C_{2}(G) K_{2}\right] /\left[C(r) K_{3}\right]$ at $\tilde{m}^{2}=0, \beta_{\tilde{g}^{2}}$ is always negative. When $N_{f}>\left[C_{2}(r) K_{1}+C_{2}(G) K_{2}\right] /\left[C(r) K_{3}\right]$ at $\tilde{m}^{2}=0$, the sign of $\beta_{\tilde{g}^{2}}$ changes at a nontrivial fixed point, $\tilde{g}^{2 *}=-\epsilon / F_{1}(0)$. The difference is that $\left[C_{2}(r) K_{1}+C_{2}(G) K_{2}\right] /\left[C(r) K_{3}\right]$ now plays the role of the lower turning point, while the larger turning point now goes to infinity.

One can see this similarity from FIG. 7. So we can use the one-loop $\beta$-function of $d$-dimensional QCD to mimic the two-loop $\beta$-function of 4-dimensional QCD. The following system will be considered:

$$
\begin{aligned}
\partial_{t} \tilde{m}^{2}= & -2 \tilde{m}^{2}\left[1+\tilde{g}^{2} C_{2}(r) K_{m}+H_{2}\left(\tilde{m}^{2}\right)\left(\tilde{g}^{2}\right)^{2}\right], \\
\partial_{t} \tilde{g}^{2}= & -2\left(\tilde{g}^{2}\right)^{2}\left[C_{2}(r) K_{1}+C_{2}(G) K_{2}-N_{f} C(r) K_{3}\right] \\
& -\tilde{g}^{2} \epsilon .
\end{aligned}
$$




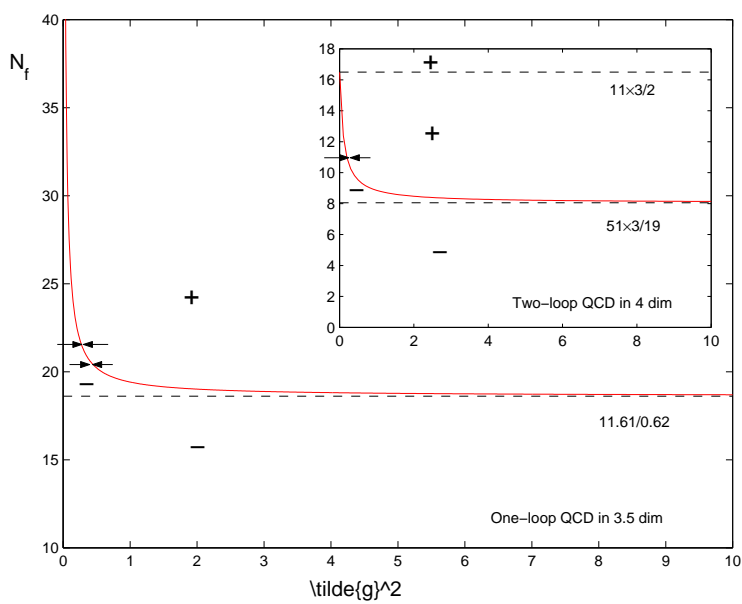

FIG. 7: The similarity between the two situations.

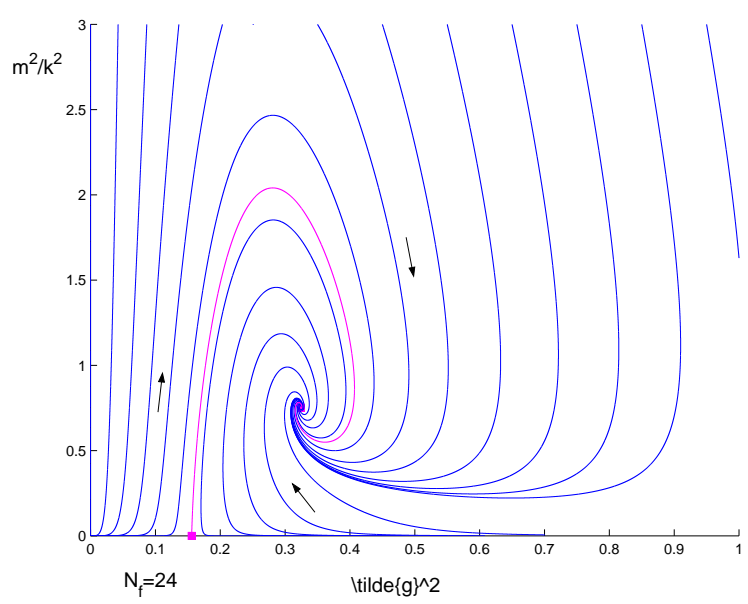

FIG. 8: IR-attractive nontrivial fixed points. The arrows point to infrared. $\left(\alpha_{i}, \beta_{i}\right)=(3,4,5,1,1,36)$.

This system deviates slightly from the one of the last section, so the flows will not be changed drastically. Since now there is a nontrivial fixed point on the axis of the coupling, there would be modifications.

Shown in FIG. 8 is the flow pattern for the situation of $d=3.5, N_{f}=24$ and $\left(\alpha_{i}, \beta_{i}\right)=(3,4,5,1,1,36)$. The pattern is much the same as that of FIG. 4 except for the fixed point on the axis. All flows are going to the nontrivial fixed point with a finite dimensionless mass, which means there is still no chiral symmetry breaking. Mapped onto two-loop $d=4$ QCD, there is no chiral symmetry breaking when $N_{f}<11 \times 3 / 2$ and larger than some critical number. So a conformal window opens up.

\section{CONCLUSION}

In conclusion, the functional renormalization group method was applied to QCD. There are variations in literature about application of functional renormalization group method to theories with both fermions and bosons. Hopefully our calculation can serve to help clarify.

A parameter was introduced to discuss the dependence of flow equations on regulators and it was shown that flow equations will be independent of regulators if this parameter is sent to infinity in the end of calculation.

Different flow patterns in the $\tilde{m}^{2}-\tilde{g}^{2}$ plane were explored. (By no means, all possible ones were reached.) One of them provides a mechanism to explain nonoccurrence of dynamical chiral symmetry breaking. However this flow pattern was obtained from flow equations that were not of QCD. To show the relevance of this mechanism to QCD, it was proved that it indeed applies to QCD, in terms of an $1 / \tilde{m}^{2}$ expansion of the fermion mass renormalization group equation of QCD. One saw that dynamical chiral symmetry breaking cannot occur if and only if the theory is attracted to an IR-attractive non-trivial fixed point of a finite dimensionless mass.

The existence of the conformal window was also discussed in the language of renormalization flow.

\section{Acknowledgement}

The work is supported in part by the National Science Foundation of China (10875103, 11135006), National Basic Research Program of China (2010CB833000).

\section{Appendix A: Details}

\section{Preparation}

If we label the fields as $(\bar{\psi}, \psi, A, \bar{c}, c)$, and let $\left(J_{\bar{\psi}}, J_{\psi}, J_{A}, J_{\bar{c}}, J_{c}\right)$ be their sources, and $\tilde{W}$ be the generation functional of connected Green functions, the twopoint connected Green function has the structure:

$$
\tilde{G}=\left(\begin{array}{ccccc}
\ldots & \tilde{G}_{\bar{\psi} \psi} & \tilde{G}_{\bar{\psi} A} & \ldots & \ldots \\
\tilde{G}_{\psi \bar{\psi}} & \ldots & \tilde{G}_{\psi A} & \ldots & \ldots \\
\tilde{G}_{A \bar{\psi}} & \tilde{G}_{A \psi} & \tilde{G}_{A A} & \tilde{G}_{A \bar{c}} & \tilde{G}_{A c} \\
\ldots & \ldots & \tilde{G}_{\bar{c} A} & \ldots & \tilde{G}_{\bar{c} c} \\
\ldots & \ldots & \tilde{G}_{c A} & \tilde{G}_{c \bar{c}} & \ldots
\end{array}\right)
$$

The terms represented by ellipsis will not be used. Other terms are defined by

$$
\tilde{G}_{\bar{\psi} \psi} \equiv \frac{\delta^{2} \tilde{W}}{\delta_{L} J_{\bar{\psi}} \delta_{R} J_{\psi}}, \quad \tilde{G}_{\bar{\psi} A} \equiv \frac{\delta^{2} \tilde{W}}{\delta_{L} J_{\bar{\psi}} \delta J_{A}}, \ldots
$$

The $\Gamma$ Hessian matrix has the structure,

$$
\Gamma^{(2)}=\left(\begin{array}{ccccc}
0 & \Gamma_{\bar{\psi} \psi}^{(2)} & \Gamma_{\bar{\psi} A}^{(2)} & 0 & 0 \\
\Gamma_{\psi \bar{\psi}}^{(2)} & 0 & \Gamma_{\psi A}^{(2)} & 0 & 0 \\
\Gamma_{A \bar{\psi}}^{(2)} & \Gamma_{A \psi}^{(2)} & \Gamma_{A A}^{(2)} & \Gamma_{A \bar{c}}^{(2)} & \Gamma_{A c}^{(2)} \\
0 & 0 & \Gamma_{\bar{c} A}^{(2)} & 0 & \Gamma_{\bar{c} c}^{(2)} \\
0 & 0 & \Gamma_{c A}^{(2)} & \Gamma_{c \bar{c}}^{(2)} & 0
\end{array}\right)
$$


Elements of the $\Gamma$ Hessian matrix are,

$$
\begin{aligned}
& \Gamma_{\bar{c} c}^{(2)} \equiv \frac{\delta^{2} \Gamma}{\delta_{L} \bar{c}^{a}(l) \delta_{R} c^{b}\left(l^{\prime}\right)} \\
& =-Z_{c} \delta^{a b} \delta_{l l^{\prime}} l^{2}-g Z_{c} \sqrt{Z_{A}} f^{a b c}\left(i l_{\kappa}\right) A_{\kappa}^{c}\left(l-l^{\prime}\right) ; \\
& \Gamma_{c \bar{c}}^{(2)} \equiv \frac{\delta^{2} \Gamma}{\delta_{L} c^{b}\left(l^{\prime}\right) \delta_{R} \bar{c}^{a}(l)} \\
& =Z_{c} \delta^{a b} \delta_{l l^{\prime}} l^{2}+g Z_{c} \sqrt{Z_{A}} f^{a b c}\left(i l_{\kappa}\right) A_{\kappa}^{c}\left(l-l^{\prime}\right) ; \\
& \Gamma_{\bar{c} A}^{(2)} \equiv \frac{\delta^{2} \Gamma}{\delta_{L} \bar{c}^{a}(l) \delta A_{\nu}^{b}\left(l^{\prime}\right)}=g Z_{c} \sqrt{Z_{A}} f^{a b c}\left(i l_{\nu}\right) c^{c}\left(l-l^{\prime}\right) \\
& \Gamma_{A \bar{c}}^{(2)} \equiv \frac{\delta^{2} \Gamma}{\delta A_{\nu}^{b}\left(-l^{\prime}\right) \delta_{R} \bar{c}^{a}(l)}=-g Z_{c} \sqrt{Z_{A}} f^{a b c}\left(i l_{\nu}\right) c^{c}\left(l+l^{\prime}\right) ; \\
& \Gamma_{A c}^{(2)} \equiv \frac{\delta^{2} \Gamma}{\delta A_{\mu}^{a}(-l) \delta_{R} c^{b}\left(l^{\prime}\right)} \\
& =g Z_{c} \sqrt{Z_{A}} f^{c a b} i\left(-l_{\mu}+l_{\mu}^{\prime}\right) \bar{c}^{c}\left(-l+l^{\prime}\right) \text {; } \\
& \Gamma_{c A}^{(2)} \equiv \frac{\delta^{2} \Gamma}{\delta_{L} c^{b}\left(l^{\prime}\right) \delta A_{\mu}^{a}(l)} \\
& =-g Z_{c} \sqrt{Z_{A}} f^{c a b} i\left(l_{\mu}+l_{\mu}^{\prime}\right) \bar{c}^{c}\left(l+l^{\prime}\right) ; \\
& \Gamma_{\bar{\psi} \psi}^{(2)} \equiv \frac{\delta^{2} \Gamma}{\delta_{L} \bar{\psi}^{a_{f}}(l) \delta_{R} \psi^{b_{f}}\left(l^{\prime}\right)} \\
& =Z_{\psi} \delta^{a_{f} b_{f}} \delta_{l l^{\prime}}(l+m)+g Z_{\psi} \sqrt{Z_{A}} \delta^{a_{f} b_{f}} \gamma^{\mu} t^{a} A_{\mu}^{a}\left(l-l^{\prime}\right) ; \\
& \Gamma_{\psi \bar{\psi}}^{(2)} \equiv \frac{\delta^{2} \Gamma}{\delta_{L} \psi^{b_{f}}\left(l^{\prime}\right) \delta_{R} \bar{\psi}^{a_{f}}(l)} \\
& =-Z_{\psi} \delta^{a_{f} b_{f}} \delta_{l l^{\prime}}\left(l^{T}+m\right)-g Z_{\psi} \sqrt{Z_{A}} \delta^{a_{f} b_{f}}\left[\gamma^{\mu} t^{a}\right]^{T} A_{\mu}^{a}\left(l-l^{\prime}\right),
\end{aligned}
$$

where $T$ means transposition;

$$
\begin{aligned}
\Gamma_{\bar{\psi} A}^{(2)} \equiv & \frac{\delta^{2} \Gamma}{\delta_{L} \bar{\psi}^{a_{f}}(l) \delta A_{\nu}^{b}\left(l^{\prime}\right)}=g Z_{\psi} \sqrt{Z_{A}} \gamma^{\nu} t^{b} \psi^{a_{f}}\left(l-l^{\prime}\right) ; \\
\Gamma_{A \bar{\psi}}^{(2)} \equiv & \frac{\delta^{2} \Gamma}{\delta A_{\nu}^{b}\left(-l^{\prime}\right) \delta_{R} \bar{\psi}^{a_{f}(l)}}=-g Z_{\psi} \sqrt{Z_{A}}\left[t^{b} \gamma^{\nu} \psi^{a_{f}}\left(l+l^{\prime}\right)\right]^{T} ; \\
\Gamma_{A \psi}^{(2)} \equiv & \frac{\delta^{2} \Gamma}{\delta A_{\mu}^{a}(-l) \delta_{R} \psi^{b_{f}}\left(l^{\prime}\right)}=g Z_{\psi} \sqrt{Z_{A}} \bar{\psi}^{b_{f}}\left(-l+l^{\prime}\right) \gamma^{\mu} t^{a} ; \\
\Gamma_{\psi A}^{(2)} \equiv & \frac{\delta^{2} \Gamma}{\delta_{L} \psi^{b_{f}}\left(l^{\prime}\right) \delta A^{a}(l)}=-g Z_{\psi} \sqrt{Z_{A}}\left[\bar{\psi}^{b_{f}}\left(l+l^{\prime}\right) \gamma^{\mu} t^{a}\right]^{T} \\
\Gamma_{A A}^{(2)} \equiv & \frac{\delta^{2} \Gamma}{\delta A_{\mu}^{a}(-l) \delta A_{\nu}^{b}\left(l^{\prime}\right)}=Z_{A} \delta^{a b} \delta^{\mu \nu} \delta_{l l^{\prime}} l^{2} \\
& +g Z_{A} \sqrt{Z_{A}} f^{a b c} i\left(2 l_{\nu}-l_{\nu}^{\prime}\right) A_{\mu}^{c}\left(l-l^{\prime}\right) \\
& +g Z_{A} \sqrt{Z_{A}} f^{a b c}(-i)\left(l_{\mu}-2 l_{\mu}^{\prime}\right) A_{\nu}^{c}\left(l-l^{\prime}\right)
\end{aligned}
$$

$$
\begin{aligned}
+g Z_{A} & \sqrt{Z_{A}} f^{a b c} \delta^{\mu \nu}(-i)\left(l_{\kappa}+l_{\kappa}^{\prime}\right) A_{\kappa}^{c}\left(l-l^{\prime}\right) \\
+g^{2} Z_{A}^{2} & \int_{q} A^{c \mu}(q) A^{d \nu}\left(l-l^{\prime}-q\right) \\
& \cdot\left(f^{e a b} f^{e c d}-f^{e a d} f^{e b c}\right) \\
+g^{2} Z_{A}^{2} & \int_{q} A^{c \lambda}(q) A^{d \lambda}\left(l-l^{\prime}-q\right) \delta_{\mu \nu} \\
& \cdot \frac{1}{2}\left(f^{e a c} f^{e b d}+f^{e a d} f^{e b c}\right) .
\end{aligned}
$$

Derivative expansions of the regulated propagators are

$$
\begin{aligned}
& \frac{1}{(l+\not p)\left(1+r_{\psi}\left((l+p)^{2} / k^{2}\right)\right)+m} \\
& \approx \frac{1}{(l+\not p)\left(1+r_{\psi}\left(l^{2} / k^{2}\right)\right)+m} \\
& =\frac{m-\not\left(1+r_{\psi}\right)}{m^{2}+l^{2}\left(1+r_{\psi}\right)^{2}}+\frac{-\not p\left(1+r_{\psi}\right)}{m^{2}+l^{2}\left(1+r_{\psi}\right)^{2}} \\
& +\left[m-\not\left(1+r_{\psi}\right)\right] \frac{-2 p \cdot l\left(1+r_{\psi}\right)^{2}}{\left[m^{2}+l^{2}\left(1+r_{\psi}\right)^{2}\right]^{2}} \\
& +\left[m-\not\left(1+r_{\psi}\right)\right] \frac{-p^{2}\left(1+r_{\psi}\right)^{2}}{\left[m^{2}+l^{2}\left(1+r_{\psi}\right)^{2}\right]^{2}} \\
& +\not p\left(1+r_{\psi}\right) \frac{2 p \cdot l\left(1+r_{\psi}\right)^{2}}{\left[m^{2}+l^{2}\left(1+r_{\psi}\right)^{2}\right]^{2}} \\
& +\left[m-\not\left(1+r_{\psi}\right)\right] \frac{4(p \cdot l)^{2}\left(1+r_{\psi}\right)^{4}}{\left[m^{2}+l^{2}\left(1+r_{\psi}\right)^{2}\right]^{3}}+O\left(p^{3} \gamma \mathrm{A} 4\right)
\end{aligned}
$$

$$
\begin{aligned}
& \frac{1}{(l+p)^{2}\left(1+r_{A}\left((l+p)^{2} / k^{2}\right)\right)} \\
& \approx \frac{1}{(l+p)^{2}\left(1+r_{A}\left(l^{2} / k^{2}\right)\right)} \\
& =\frac{1}{l^{2}\left(1+r_{A}\right)}-\frac{2 p \cdot l}{\left(l^{2}\right)^{2}\left(1+r_{A}\right)} \\
& -\frac{p^{2}}{\left(l^{2}\right)^{2}\left(1+r_{A}\right)}+\frac{4(p \cdot l)^{2}}{\left(l^{2}\right)^{3}\left(1+r_{A}\right)}+O\left(p^{3}\right) .
\end{aligned}
$$

The $\partial_{t}$ derivatives of the regulated propagators are

$$
\begin{aligned}
& \frac{1}{\not\left(1+r_{\psi}\right)+m}\left(\partial_{t} r_{\psi}\right) l \frac{1}{\lambda\left(1+r_{\psi}\right)+m} \\
& =\left(\partial_{t} r_{\psi}\right) \frac{\left[m^{2}-l^{2}\left(1+r_{\psi}\right)^{2} l l+2 m l^{2}\left(1+r_{\psi}\right)\right.}{\left[m^{2}+l^{2}\left(1+r_{\psi}\right)^{2}\right]^{2}} \\
& \equiv F_{1} l+F_{2} m .
\end{aligned}
$$




\section{Fermion propagator}

To get the flow equation for the fermion propagator, we encounter

$$
\begin{aligned}
& \frac{1}{2} \operatorname{Tr}\left(Z_{A} \partial_{t} r_{A} l^{2}\right) \frac{1}{Z_{A} l^{2}\left(1+r_{A}\right)} g^{2} Z_{\psi} Z_{A} \bar{\psi}^{a_{f}}\left(-l+l^{\prime \prime}\right) \gamma^{\mu} t^{a} \frac{1}{l^{\prime \prime}\left(1+r_{\psi}\right)+m} \gamma^{\nu} t^{b} \psi^{a_{f}}\left(l^{\prime \prime}-l^{\prime}\right) \frac{1}{Z_{A} l^{\prime 2}\left(1+r_{A}\right)} \\
& -\frac{1}{2} \operatorname{Tr}\left(Z_{A} \partial_{t} r_{A} l^{2}\right) \frac{1}{Z_{A} l^{2}\left(1+r_{A}\right)} g^{2} Z_{\psi} Z_{A}\left[t^{b} \gamma^{\nu} \psi^{a_{f}}\left(l^{\prime \prime}+l\right)\right]^{T} \frac{1}{l^{\prime \prime T}\left(1+r_{\psi}\right)+m}\left[\bar{\psi}^{a_{f}}\left(l^{\prime}+l^{\prime \prime}\right) \gamma^{\mu} t^{a}\right]^{T} \frac{1}{Z_{A} l^{\prime 2}\left(1+r_{A}\right)} \\
& -\operatorname{Tr}\left(Z_{\psi} \partial_{t} r_{\psi} l\right) \frac{1 / Z_{\psi}}{\lambda\left(1+r_{\psi}\right)+m} g^{2} Z_{\psi}^{2} Z_{A} \gamma^{\mu} t^{a} \psi^{a_{f}}\left(l-l^{\prime \prime}\right) \frac{1}{Z_{A} l^{\prime \prime 2}\left(1+r_{A}\right)} \bar{\psi}^{b_{f}}\left(-l^{\prime \prime}+l^{\prime}\right) \gamma^{\mu} t^{a} \frac{1 / Z_{\psi}}{l^{\prime}\left(1+r_{\psi}\right)+m} \\
& =g^{2} Z_{\psi} C_{2}(r)(-d) \int_{p} \bar{\psi}^{a_{f}}(p) \psi^{a_{f}}(p) m \cdot \int_{l}\left\{\frac{B}{m^{2}+l^{2}\left(1+r_{\psi}\right)^{2}}+\frac{F_{2}}{l^{2}\left(1+r_{A}\right)}\right\} \\
& +g^{2} Z_{\psi} C_{2}(r)(d-2) \int_{p} \bar{\psi}^{a_{f}}(p) p \psi^{a_{f}}(p) \cdot \int_{l}\left\{\frac{-B\left(1+r_{\psi}\right)}{m^{2}+l^{2}\left(1+r_{\psi}\right)^{2}}+\frac{2 B\left(1+r_{\psi}\right)^{3} l^{2} / d}{\left[m^{2}+l^{2}\left(1+r_{\psi}\right)^{2}\right]^{2}}+\frac{2 F_{1} / d}{l^{2}\left(1+r_{A}\right)}\right\} \\
& + \text { higher order terms. }
\end{aligned}
$$

The flow equations for $m$ and $Z_{\psi}$ can be read off as

$$
\begin{aligned}
\frac{\partial_{t} m}{m} & =-\tilde{g}^{2} C_{2}(r)\left[d J\left(1,4,1 ; \tilde{m}^{2}\right)+d J\left(0,2,2 ; \tilde{m}^{2}\right)-\frac{(d-1)(d-2)}{d} J\left(1,3,1 ; \tilde{m}^{2}\right)\right], \\
\frac{\partial_{t} Z_{\psi}}{Z_{\psi}} & =-\tilde{g}^{2} C_{2}(r)\left[\frac{(d-1)(d-2)}{d} J\left(1,3,1 ; \tilde{m}^{2}\right)\right] .
\end{aligned}
$$

\section{Gluon propagator}

Here one encounters

$$
\operatorname{Tr}\left(Z_{A} \partial_{t} r_{A} l^{2}\right) \frac{1}{Z_{A} l^{2}\left(1+r_{A}\right)} g^{2} Z_{A}^{2}\{\cdots\} \frac{1}{Z_{A} l^{\prime 2}\left(1+r_{A}\right)}=0
$$

Here $g^{2} Z_{A}^{2}\{\ldots\}$ indicates terms in $\Gamma_{A A}^{(2)}$ that come from the 4-gluon vertex.

To get the flow equation for the gluon propagator, we encounter

$$
\begin{aligned}
& \frac{1}{2} \operatorname{Tr}\left(Z_{A} \partial_{t} r_{A} l^{2}\right) \frac{1}{Z_{A} l^{2}\left(1+r_{A}\right)} g Z_{A} \sqrt{Z_{A}}\{\ldots\} \frac{1}{Z_{A} l^{\prime \prime 2}\left(1+r_{A}\right)} g Z_{A} \sqrt{Z_{A}}\{\ldots\} \frac{1}{Z_{A} l^{\prime 2}\left(1+r_{A}\right)} \\
& -\operatorname{Tr}\left(-Z_{c} \partial_{t} r_{A} l^{2}\right) \frac{-1}{Z_{c} l^{2}\left(1+r_{A}\right)} g Z_{c} \sqrt{Z_{A}} f^{a c e}\left(i l_{\kappa}\right) A_{\kappa}^{e}\left(l-l^{\prime \prime}\right) \frac{-1}{Z_{c} l^{\prime \prime 2}\left(1+r_{A}\right)} g Z_{c} \sqrt{Z_{A}} f^{c b e^{\prime}}\left(i l_{\lambda}^{\prime \prime}\right) A_{\lambda}^{e^{\prime}}\left(l^{\prime \prime}-l^{\prime}\right) \frac{-1}{Z_{c} l^{\prime 2}\left(1+r_{A}\right)} \\
& -\operatorname{Tr}\left(Z_{\psi} \partial_{t} r_{\psi} l\right) \frac{1 / Z_{\psi}}{\lambda\left(1+r_{\psi}\right)+m} g Z_{\psi} \sqrt{Z_{A}} \gamma^{\mu} t^{a} A_{\mu}^{a}\left(l-l^{\prime \prime}\right) \frac{1 / Z_{\psi}}{\ell^{\prime \prime}\left(1+r_{\psi}\right)+m} g Z_{\psi} \sqrt{Z_{A}} \gamma^{\nu} t^{b} A_{\nu}^{b}\left(l^{\prime \prime}-l^{\prime}\right) \frac{1 / Z_{\psi}}{\lambda^{\prime}\left(1+r_{\psi}\right)+m} \\
& =g^{2} Z_{A} C_{2}(G) \int_{p} A_{\mu}^{e}(-p)\left(\delta_{\mu \nu} p^{2}-p_{\mu} p_{\nu}\right) A_{\nu}^{e}(p) \cdot \int_{l} \frac{-B}{l^{2}\left(1+r_{A}\right)}\left[\frac{16 d-32}{d(d+2)}+\frac{d-14}{2}+\frac{8}{d}\right] \\
& -g^{2} Z_{A} N_{f} C(r) \int_{p} A_{\mu}^{e}(-p)\left(\delta_{\mu \nu} p^{2}-p_{\mu} p_{\nu}\right) A_{\nu}^{e}(p) \cdot \int_{l}\left\{\frac{F_{1}\left(1+r_{\psi}\right)^{3} l^{2}}{\left[m^{2}+l^{2}\left(1+r_{\psi}\right)^{2}\right]^{2}} \frac{-16}{d}+\frac{F_{1}\left(1+r_{\psi}\right)^{5}\left(l^{2}\right)^{2}}{\left[m^{2}+l^{2}\left(1+r_{\psi}\right)^{2}\right]^{3}} \frac{64}{d(d+2)}\right\}
\end{aligned}
$$

+ higher order terms and gauge symmetry breaking terms.

Here $g Z_{A} \sqrt{Z_{A}}\{\ldots\}$ indicates terms in $\Gamma_{A A}^{(2)}$ that come from the 3 -gluon vertex.

The flow equation for $Z_{A}$ can be read off as

$$
\begin{aligned}
\frac{1}{2} \frac{\partial_{t} Z_{A}}{Z_{A}} & =-\tilde{g}^{2} C_{2}(G)\left[\frac{16 d-32}{d(d+2)}+\frac{d-14}{2}+\frac{8}{d}\right] J\left(2,6,0 ; \tilde{m}^{2}\right) \\
& -\tilde{g}^{2} N_{f} C(r)\left[-\frac{8}{d} J\left(-1,-2,3 ; \tilde{m}^{2}\right)+\frac{16(d+4)}{d(d+2)} J\left(-2,-4,4 ; \tilde{m}^{2}\right)-\frac{64}{d(d+2)} J\left(-3,-6,5 ; \tilde{m}^{2}\right)\right]
\end{aligned}
$$




\section{Fermion-gluon vertex}

The flow equation for the coupling can be derived from the renormalized fermion-gluon vertex.

$$
\begin{aligned}
& \frac{1}{2} \operatorname{Tr}\left(Z_{A} \partial_{t} r_{A} l^{2}\right) \frac{-1}{Z_{A} l^{2}\left(1+r_{A}\right)} g^{2} Z_{\psi} Z_{A} \bar{\psi}^{a_{f}}\left(-l+l^{\prime \prime}\right) \gamma^{\mu} t^{a} \frac{1}{\ell^{\prime \prime}\left(1+r_{\psi}\right)+m} g \sqrt{Z_{A}} A_{\rho}^{e}\left(l^{\prime \prime}-l^{\prime \prime \prime}\right) \gamma^{\rho} t^{e} \frac{1}{\ell^{\prime \prime \prime}\left(1+r_{\psi}\right)+m} \\
& \cdot \gamma^{\nu} t^{b} \psi^{a_{f}}\left(l^{\prime \prime \prime}-l^{\prime}\right) \frac{1}{Z_{A} l^{\prime 2}\left(1+r_{A}\right)} \\
& -\frac{1}{2} \operatorname{Tr}\left(Z_{A} \partial_{t} r_{A} l^{2}\right) \frac{-1}{Z_{A} l^{2}\left(1+r_{A}\right)} g^{2} Z_{\psi} Z_{A}\left[t^{b} \gamma^{\nu} \psi^{a_{f}}\left(l^{\prime \prime}+l\right)\right]^{T} \frac{1}{l^{\prime \prime T}\left(1+r_{\psi}\right)+m} g \sqrt{Z_{A}} A_{\rho}^{e}\left(-l^{\prime \prime}+l^{\prime \prime \prime}\right)\left[\gamma^{\rho} t^{e}\right]^{T} \frac{1}{l^{\prime \prime \prime T}}\left(1+r_{\psi}\right)+m \\
& \cdot\left[\bar{\psi}^{a_{f}}\left(l^{\prime}+l^{\prime \prime \prime}\right) \gamma^{\mu} t^{a}\right]^{T} \frac{1}{Z_{A} l^{\prime 2}\left(1+r_{A}\right)} \\
& -\operatorname{Tr}\left(Z_{\psi} \partial_{t} r_{\psi} l\right) \frac{1 / Z_{\psi}}{l\left(1+r_{\psi}\right)+m} g Z_{\psi} \sqrt{Z_{A}} A_{\rho}^{e}\left(l-l^{\prime \prime}\right) \gamma^{\rho} t^{e} \frac{1 / Z_{\psi}}{l^{\prime \prime}\left(1+r_{\psi}\right)+m} \\
& \cdot\left(-g Z_{\psi} \sqrt{Z_{A}}\right) \gamma^{\mu} t^{a} \psi^{a_{f}}\left(l^{\prime \prime}-l^{\prime \prime \prime}\right) \frac{1}{Z_{A} l^{\prime \prime \prime 2}\left(1+r_{A}\right)}\left(g Z_{\psi} \sqrt{Z_{A}}\right) \bar{\psi}^{b_{f}}\left(-l^{\prime \prime \prime}+l^{\prime}\right) \gamma^{\mu} t^{a} \frac{1 / Z_{\psi}}{l^{\prime \prime}\left(1+r_{\psi}\right)+m} \\
& -\operatorname{Tr}\left(Z_{\psi} \partial_{t} r_{\psi} l\right) \frac{1 / Z_{\psi}}{\lambda\left(1+r_{\psi}\right)+m}\left(-g Z_{\psi} \sqrt{Z_{A}}\right) \gamma^{\mu} t^{a} \psi^{a_{f}}\left(l-l^{\prime \prime}\right) \frac{1}{Z_{A} l^{\prime \prime 2}\left(1+r_{A}\right)}\left(g Z_{\psi} \sqrt{Z_{A}}\right) \bar{\psi}^{b_{f}}\left(-l^{\prime \prime}+l^{\prime \prime \prime}\right) \gamma^{\mu} t^{a} \\
& \cdot \frac{1 / Z_{\psi}}{\ell^{\prime \prime \prime}\left(1+r_{\psi}\right)+m} g Z_{\psi} \sqrt{Z_{A}} A_{\rho}^{e}\left(l^{\prime \prime \prime}-l^{\prime}\right) \gamma^{\rho} t^{e} \frac{1 / Z_{\psi}}{l^{\prime}\left(1+r_{\psi}\right)+m} \\
& +\frac{1}{2} \operatorname{Tr}\left(Z_{A} \partial_{t} r_{A} l^{2}\right) \frac{1}{Z_{A} l^{2}\left(1+r_{A}\right)}\left(-g^{2} Z_{\psi} \sqrt{Z_{A}}\right) \bar{\psi}^{a_{f}}\left(-l+l^{\prime \prime}\right) \gamma^{\mu} t^{a} \frac{1}{l^{\prime \prime}\left(1+r_{\psi}\right)+m} \gamma^{\rho} t^{c} \psi^{a_{f}}\left(l^{\prime \prime}-l^{\prime \prime \prime}\right) \\
& \cdot \frac{1}{Z_{A} l^{\prime \prime \prime 2}\left(1+r_{A}\right)} g Z_{A} \sqrt{Z_{A}}\{\ldots\}_{l^{\prime \prime \prime} l^{\prime}}^{c b, \rho \nu} \frac{1}{Z_{A} l^{\prime 2}\left(1+r_{A}\right)} \\
& +\frac{1}{2} \operatorname{Tr}\left(Z_{A} \partial_{t} r_{A} l^{2}\right) \frac{1}{Z_{A} l^{2}\left(1+r_{A}\right)} g Z_{A} \sqrt{Z_{A}}\{\ldots\}_{l l^{\prime \prime}}^{a c, \mu \rho} \frac{1}{Z_{A} l^{\prime \prime 2}\left(1+r_{A}\right)} \\
& \cdot\left(-g^{2} Z_{\psi} \sqrt{Z_{A}}\right) \bar{\psi}^{a_{f}}\left(-l^{\prime \prime}+l^{\prime \prime \prime}\right) \gamma^{\rho} t^{c} \frac{1}{l^{\prime \prime \prime}\left(1+r_{\psi}\right)+m} \gamma^{\nu} t^{b} \psi^{a_{f}}\left(l^{\prime \prime \prime}-l^{\prime}\right) \frac{1}{Z_{A} l^{\prime 2}\left(1+r_{A}\right)} \\
& -\frac{1}{2} \operatorname{Tr}\left(Z_{A} \partial_{t} r_{A} l^{2}\right) \frac{1}{Z_{A} l^{2}\left(1+r_{A}\right)}\left(-g^{2} Z_{\psi} \sqrt{Z_{A}}\right)\left[t^{a} \gamma^{\mu} \psi^{a_{f}}\left(l^{\prime \prime}+l\right)\right]^{T} \frac{1}{l^{\prime \prime T}\left(1+r_{\psi}\right)+m}\left[\bar{\psi}^{a_{f}}\left(l^{\prime \prime \prime}+l^{\prime \prime}\right) \gamma^{\rho} t^{c}\right]^{T} \\
& \cdot \frac{1}{Z_{A} l^{\prime \prime \prime 2}\left(1+r_{A}\right)} g Z_{A} \sqrt{Z_{A}}\{\ldots\}_{l^{\prime \prime \prime} l^{\prime}}^{c b, \rho \nu} \frac{1}{Z_{A} l^{\prime 2}\left(1+r_{A}\right)} \\
& -\frac{1}{2} \operatorname{Tr}\left(Z_{A} \partial_{t} r_{A} l^{2}\right) \frac{1}{Z_{A} l^{2}\left(1+r_{A}\right)} g Z_{A} \sqrt{Z_{A}}\{\ldots\}_{l l^{\prime \prime}}^{a c, \mu \rho} \frac{1}{Z_{A} l^{\prime \prime 2}\left(1+r_{A}\right)} \\
& \cdot\left(-g^{2} Z_{\psi} \sqrt{Z_{A}}\right)\left[t^{c} \gamma^{\rho} \psi^{a_{f}}\left(l^{\prime \prime \prime}+l^{\prime \prime}\right)\right]^{T} \frac{1}{l^{\prime \prime \prime T}\left(1+r_{\psi}\right)+m}\left[\bar{\psi}^{a_{f}}\left(l^{\prime}+l^{\prime \prime \prime}\right) \gamma^{\nu} t^{b}\right]^{T} \frac{1}{Z_{A} l^{\prime 2}\left(1+r_{A}\right)} \\
& -\operatorname{Tr}\left(Z_{\psi} \partial_{t} r_{\psi} l\right) \frac{-1 / Z_{\psi}}{\lambda\left(1+r_{\psi}\right)+m}\left(-g Z_{\psi} \sqrt{Z_{A}}\right) \gamma^{\nu} t^{b} \psi^{a_{f}}\left(l-l^{\prime \prime}\right) \frac{-1}{Z_{A} l^{\prime \prime 2}\left(1+r_{A}\right)} g Z_{\psi} \sqrt{Z_{A}}\{\ldots\}_{l^{\prime \prime} l^{\prime \prime \prime}}^{\nu \mu, b a} \frac{1}{Z_{A} l^{\prime \prime \prime 2}\left(1+r_{A}\right)} \\
& g Z_{\psi} \sqrt{Z_{A}} \bar{\psi}^{b_{f}}\left(l^{\prime \prime \prime}-l^{\prime}\right) \gamma^{\mu} t^{a} \frac{1 / Z_{\psi}}{l^{\prime}\left(1+r_{\psi}\right)+m} \\
& =g^{3} Z_{\psi} \sqrt{Z_{A}} \int_{p} \int_{p^{\prime}} \bar{\psi}^{a_{f}}(p) \gamma^{\mu} t^{a} A_{\mu}^{a}\left(p-p^{\prime}\right) \psi^{a_{f}}\left(p^{\prime}\right) \\
& \cdot\left\{-\left[C_{2}(r)-\frac{1}{2} C_{2}(G)\right] \int_{l} B \frac{l^{2}\left(1+r_{\psi}\right)^{2}(d-2)^{2} / d+m^{2}(d-2)}{\left[m^{2}+l^{2}\left(1+r_{\psi}\right)^{2}\right]^{2}}\right. \\
& -2\left[C_{2}(r)-\frac{1}{2} C_{2}(G)\right] \int_{l} \frac{-F_{1} l^{2}\left(1+r_{\psi}\right)(d-2)^{2} / d+F_{2} m^{2}(d-2)}{m^{2}+l^{2}\left(1+r_{\psi}\right)^{2}} \frac{1}{l^{2}\left(1+r_{A}\right)} \\
& \left.-C_{2}(G) \int_{l} \frac{B}{l^{2}\left(1+r_{A}\right)} \frac{4 l^{2}\left(1+r_{\psi}\right)}{m^{2}+l^{2}\left(1+r_{\psi}\right)^{2}} \frac{d-1}{d}-\frac{1}{2} C_{2}(G) \int_{l} \frac{1}{\left[l^{2}\left(1+r_{A}\right)\right]^{2}}\left(-4 l^{2} F_{1}\right) \frac{d-1}{d}\right\}
\end{aligned}
$$

+ higher order terms. 
Then the flow equation for $g Z_{\psi} \sqrt{Z_{A}}$ can be read off as

$$
\begin{aligned}
\frac{\partial_{t}\left(g Z_{\psi} \sqrt{Z_{A}}\right)}{g Z_{\psi} \sqrt{Z_{A}}} & =-\tilde{g}^{2}\left[C_{2}(r)-\frac{1}{2} C_{2}(G)\right]\left[(d-2) J\left(1,4,1 ; \tilde{m}^{2}\right)+(d-2) J\left(0,2,2 ; \tilde{m}^{2}\right)-\frac{4(d-2)}{d} J\left(-1,0,3 ; \tilde{m}^{2}\right)\right] \\
& -\frac{1}{2} \tilde{g}^{2} C_{2}(G)\left[\frac{6 d-6}{d} J\left(1,5,1 ; \tilde{m}^{2}\right)+\frac{4 d-4}{d} J\left(0,3,2 ; \tilde{m}^{2}\right)\right]
\end{aligned}
$$

\section{Appendix B: Some identities}

With the convention $\left\{\gamma^{\mu}, \gamma^{\nu}\right\}=-2 \delta^{\mu \nu} I_{4 \times 4}$ and $d$ dimensional Euclidian spacetime,

$$
\begin{gathered}
\gamma^{\mu} \gamma^{\mu}=(-d) I_{4 \times 4} . \\
\gamma^{\mu} \gamma^{\rho} \gamma^{\mu}=(d-2) \gamma^{\rho} . \\
\gamma^{\mu} \mu \gamma^{\rho} \not \gamma^{\mu}=(d-2) l^{2} \gamma^{\rho}-(d-2) 2 \mu l_{\rho} . \\
\mu l=\left(-l^{2}\right) I_{4 \times 4} . \\
\not \gamma^{\rho} l=l^{2} \gamma^{\rho}-2 \mu l_{\rho} .
\end{gathered}
$$

$$
\operatorname{Tr}\left(\gamma^{\mu} \gamma^{\nu}\right)=-4 \delta^{\mu \nu}
$$

Color matrix products:

$$
\begin{gathered}
t^{a} t^{b} t^{a}=\left[C_{2}(r)-\frac{1}{2} C_{2}(G)\right] t^{b} \\
t^{a} t^{b} f^{a b c}=\frac{1}{2} i C_{2}(G) t^{c}
\end{gathered}
$$

The following expansion will be used frequently,

$$
\frac{1}{A+\epsilon}=\frac{1}{A}-\frac{1}{A} \epsilon \frac{1}{A}+\frac{1}{A} \epsilon \frac{1}{A} \epsilon \frac{1}{A}-\ldots
$$

[1] J. Polchinski, Nucl. Phys. B 231: 269 (1984)

[2] C. Wetterich, Phys. Lett. B 301: 90 (1993)

[3] O. J. Rosten, arXiv:1003.1366

[4] H. Gies, arXiv:hep-ph/0611146

[5] Holger Gies, Joerg Jaeckel, Phys. Rev. Lett. 93, 110405 (2004) (arXiv:hep-ph/0405183v1)

[6] Holger Gies, Phys. Rev. D 66, 025006 (2002)

[7] C. S. Fischer, H. Gies, arXiv:hep-ph/0408089v2

[8] D. Litim, C. Wetterich, N. Tetradis, Mod. Phys. Lett. A12 (1997) 2287-2308, arXiv:hep-ph/9407267 N. Tetradis, Nucl. Phys. B488 (1997) 92-140, arXiv:hep-ph/9608272 J. Berges, N. Tetradis, C. Wetterich, Phys. Rept. 363, 223-386 (2002), arXiv:hep-ph/0005122

[9] S. Yoshida and T. Fukui, Phys. Rev. E 72, 046136 (2005)

[10] D. F. Litim, Phys. Rev. D 76. 105001 (2007)

[11] M. M. Scherer, S. Floerchinger and H. Gies, Phil. Trans. R. Soc. A 369, 2779-2799 (2011)

[12] M. C. Ogilvie, Phil. Trans. R. Soc. A 369, 2718-2734 (2011)

[13] M. C. Birse, Phil. Trans. R. Soc. A 369, 2662-2678 (2010)

[14] Y. Meurice, R. Perry and S.-W. Tsai, Phil. Trans. R. Soc. A 369, 2602-2611 (2011)

[15] S. Nagy, I. Nandori, J. Polonyi, K. Sailer, Phys. Rev. Lett. 102: 241603, 2009 (arXiv:0904.3689v1)

[16] I. Nandori, S. Nagy, K. Sailer and A. Trombettoni, Phys. Rev. D 80, 025008 (2009) (arXiv:0903.5524)

[17] Sergej Moroz, Richard Schmidt, Annals Phys. 325: 491513, 2010 (arXiv:0909.3477v3)

[18] S. Nagy, K. Sailer, Annals Phys. 326: 1839-1876, 2011 (arXiv:1009.4041)
[19] Daniel F. Litim and Dario Zappala, Phys. Rev. D 83: 085009 (2011)

[20] A. Codello, R. Percacci, C. Rahmede, Annals Phys. 324: 414-469, 2009 (arXiv:0805.2909)

[21] F. Saueressig, K. Groh, S. Rechenberger, O. Zanusso, arXiv:1111.1743 1

[22] I. Nandori, Phys. Rev. D 84: 065024, 2011 (arXiv:1008.2934 v3)

[23] T. Appelquist, G. T. Fleming, and E. T. Neil, Phys. Rev. Lett. 100, 171607 (2008); Phys. Rev. D 79, 076010 (2009)

[24] A. Deuzeman, M. P. Lombardo, and E. Pallante, Phys. Rev. D 82, 074503 (2010)

[25] T. Appelquist, etc. (LSD Collaboration), Phys. Rev. Lett. 104, 071601 (2010)

[26] Z. Fodor, K. Holland, J. Kuti, D. Nogradi, C. Schroeder, Phys. Lett. B 703 (2011) 348-358

[27] T. Appelquist, G. T. Fleming, M. F. Lin, E. T. Neil, and D. A. Schaich, Phys. Rev. D 84, 054501 (2011)

[28] T. A. Ryttov and F. Sannino, Phys. Rev. D 78, 065001 (2008)

[29] D. D. Dietrich, Phys. Rev. D 80, 065032 (2009); Phys. Rev. D 82, 065007 (2010)

[30] J. Braun, C. S. Fischer, and Holger Gies, Phys. Rev. D 84, 034045 (2011)

[31] M. E. Peskin, D. V. Schroeder, An Introduction to Quantum Field theory, Addison-Wesley Publishing Company, 1995

[32] Lay-Nam Chang, Ngee-Pong Chang, Phys. Rev. D 29, 312-317 (1984) 